\title{
Avaliação da coloração e rugosidade do esmalte dental submetido ao clareamento sob desafio de envelhecimento em bebidas fitness
}

Dental enamel's coloration and rugosity evaluation under aging challenges in fitness drinks when submitted to whitening methods Evaluación de la coloración y rugosidad del esmalte dental sometido al claramiento bajo desafío de envejecimiento en bebidas fitness

\author{
Daniela Lopes das Silva AMORIELI ${ }^{1}$ \\ Murilo Rafael Pereira LOPES ${ }^{1}$ \\ Rafaela Caroline da SILVA ${ }^{1}$ \\ Eliane Cristina Gava PIZI $^{\mathbf{1}}$ \\ Rosana Leal do PRADO ${ }^{1}$ \\ Anderson CATELAN ${ }^{1}$ \\ Cristina Atsumi KUBA ${ }^{2}$ \\ Paulo Henrique dos SANTOS ${ }^{3}$ \\ Larissa Sgarbosa de Araújo MATUDA ${ }^{1}$
}

${ }^{1}$ Programa de Graduação em Odontologia- Clínica Odontológica, Unoeste Univ. do Oeste Paulista, 19050-920, Presidente Prudente - SP, Brasil

${ }^{2}$ Programa de Graduação em Nutrição, Unoeste Univ. do Oeste Paulista, 19050-920, Presidente Prudente - SP, Brasil

${ }^{3}$ Universidade Estadual Paulista (Unesp), Faculdade de Odontologia, Araçatuba 16015-050 Araçatuba-SP, Brasil

\section{Resumo}

O clareamento dental tem sido uma opção conservadora para o tratamento estético dos dentes. A procura pelo procedimento tornou-se popular, sendo considerado ainda um procedimento minimamente invasivo, seguro e eficaz quando feito corretamente e acompanhado por um profissional habilitado. Esse procedimento poderá mudar significativamente a aparência dos dentes, tornando-a agradável. O propósito deste estudo foi avaliar o efeito do clareamento de consultório do esmalte dental sobre sua alteração de cor e a rugosidade superficial após o envelhecimento artificial embebidas fitness. 100 dentes bovinos foram submetidos a uma análise cromática inicial, por meio de um Espectrofotômetro de Reflexão Ultravioleta Visível*,Modelo* VITA Easyshade ${ }^{\circledR}$ Compact, com a avaliação de cor calculada através do Sistema CIE L*a*b*. A análise de rugosidade superficial $(\mathrm{Ra})$ dos blocos de esmalte foi realizada em rugosímetro HommelEtamic W10 (JENOPTIK Industrial Metrology Germany GmbH). Após as análises iniciais os dentes foram divididos em10 grupos de estudo $(\mathrm{n}=10)$ - 50 amostras clareadas e 50 amostras não clareadas (3sessões-1/semana)sendo imersas em sucos detox de açai (DTXAc), rosa (DTXRs), verde (DTXV), amarelo (DTXAm) e água mineral(A) por 1 hora/ dia. A alteração de $\operatorname{cor}(\Delta \mathrm{E})$ e rugosidade $(\mathrm{Ra})$ foram calculados.Não foi observada diferença estatisticamente significante na porcentagem de aumento da rugosidade das amostras. Apresentaram maior alteração de cor, as amostras clareadas que foram submetidas a envelhecimento artificial em DTXAm e DTXRs. Entre os grupos que não foram clareados, os Sucos DTXRs, de DTXAc e DTXV apresentaram maior alteração de cor. O uso de Sucos DTXRs e DTXAm entre as sessões de clareamento de consultório resultou em maior alteração de cor.

Descritores: Esmalte Dentário; Clareamento Dental; Clareadores Dentários.

\section{Abstract}

Dental whitening has been a conservative option for the aesthetic treatment of the teeth. The search for this procedure has become popular and it's still considered a minimally invasive, safe and effective option for those how do it correctly with a qualified professional. This procedure can significantly change the appearance of the teeth, making it more pleasant. The purpose of this study was to evaluate the effect of dental enamel whitening on its color change and surface roughness after artificial aging in fitness drinks. 100 bovine teeth were submitted to an initial chromatic analysis through a VITA Easyshade ${ }^{\circledR}$ Compact Visible Ultraviolet Reflection Spectrophotometer *, with a color evaluation calculated through the CIE System L $* a * b *$. The surface roughness analysis (Ra) of the enamel blocks was performed on a Hommel Etamic W10 rugosimeter (JENOPTIK Industrial Metrology Germany GmbH). After the initial analyzes, the teeth were divided into 10 study groups $(\mathrm{n}=10)-50$ whitened samples and 50 unwhitened samples (3 sessions-1/ week) - being immersed in acai berry (DTXAc), rose (DTXR), green (DTXG), yellow (DTXY) and distilled water $(\mathrm{W})$ for 1 hour / day. Color change $(\Delta \mathrm{E})$ and roughness (Ra) were calculated. No statistically significant difference was observed in the roughness parcentage value of the samples. There was a higher color change on the whitened samples that were submitted to artificial aging in DTXY and DTXR. Among the groups that were not whitened, the DTXR, DTXAc and DTXG Juices presented greater color change. The use of DTXR and DTXY between the office treatment sessions resulted in greater color change.

Descriptors: Dental Enamel; Tooth Bleaching; Tooth Bleaching Agents.

\section{Resumen}

El blanqueamiento dental ha sido una opción conservadora para el tratamiento estético de los dientes. La demanda por el procedimiento se ha vuelto popular, siendo considerado un procedimiento mínimamente invasivo, seguro y eficaz cuando es hecho correctamente y acompañado por un profesional habilitado. Este procedimiento puede cambiar significativamente la apariencia de los dientes, haciéndola agradable. El propósito de este estudio fue evaluar el efecto del blanqueamiento de consultorio del esmalte dental sobre su alteración de color y la rugosidad superficial después del envejecimiento artificial empapado de fitness. 100 dientes bovinos fueron sometidos a un análisis cromático inicial, por medio de un Espectrofotómetro de Reflexión Ultravioleta Visible *, Modelo * VITA Easyshade ${ }^{\circledR}$ Compact, con la evaluación de color calculada a través del Sistema CIE L * a * b *. El análisis de rugosidad superficial $(\mathrm{Ra})$ de los bloques de esmalte fue realizado en rugosímetro HommelEtamic W10 (JENOPTIK Industrial Metrology Germany GmbH). $(\mathrm{N}=10)$ - 50 muestras blanqueadas y 50 muestras no blanqueadas (3ses-1/ semana) -sin sumergidas en jugos detox de acai (DTXAc), rosa (DTXR), verde (DTXV), amarillo (amarillo) DTXAm) y el águila (A) durante 1 hora / día. La alteración de color $(\Delta \mathrm{E})$ y rugosidad $(\mathrm{Ra})$ fueron calculados. No se observó diferencia estadísticamente significativa en el porcentaje de aumento de la rugosidad de las muestras. Se presentó mayor alteración de color, las muestras blanqueadas que fueron sometidas a envejecimiento artificial en DTXAm y DTXR. Entre los grupos que no fueron blanqueados, los jugos DTXR, de DTXAc y DTXV presentaron mayor alteración de color. El uso de jugos DTXR y DTXAm entre las sesiones de blanqueamiento de consultorio resultó en mayor alteración de color.

Descriptores: Esmalte Dental; Blanqueamiento dental; Careadores Dentales.

\section{INTRODUÇÃO}

Com o aumento cada vez mais crescente da busca da estética pelos pacientes, o clareamento dental tem sido uma opção conservadora para o tratamento estético dos dentes ${ }^{1-3}$. Os produtos clareadores à base de peróxido de carbamida, comumente encontrados no mercado odontológico na forma de gel, possuem em sua composição o agente peróxido de hidrogênio que é fundamental para a ocorrência do clareamento de estrutura dental ${ }^{4}$.

A procura pelo procedimento tornou-se popular, sendo considerado ainda um procedimento minimamente invasivo, seguro e eficaz quando feito corretamente e acompanhado por um profissional habilitado ${ }^{5}$. Contudo, apesar de sua popularidade, tem se discutido que as técnicas podem promover, em estudos in vitro, alterações da micromorfologia 
superficial dos tecidos dentários que seriam responsáveis por alterações na rugosidade superficial ${ }^{6}$ microdureza e cor ${ }^{5}$ do esmalte clareado.

O padrão de estética é subjetivo e varia dependendo de certos conceitos raciais, étnicos e fatores individuais. Cada civilização desenvolve seu próprio conceito de estética, no entanto, dentes com tonalidades mais claras, bem alinhados e contornados, têm sido uma exigência frequente. A cor dos dentes é um dos fatores mais importantes para a manutenção do equilíbrio estético do sorriso, pois, um dente escurecido, interfere negativamente na aparência do sorriso, podendo gerar desordens psicológicas e até sociais nos indivíduos, sendo a desarmonia mais rapidamente notada pelas pessoas ${ }^{7}$.

Independente do fator etiológico do escurecimento dentário, o clareamento é a primeira alternativa de tratamento para esses dentes. Esse procedimento poderá mudar significativamente a aparência dos dentes, tornando-a agradável. A clareação dentária tem sido uma alternativa conservadora para a restauração da estética em dentes polpados e despolpados, escurecidos e manchados, sendo um dos procedimentos estéticos mais procurados pelos pacientes ${ }^{7}$.

A alteração cromática dos dentes ocorre devido a fatores extrínsecos e intrínsecos. As alterações de origem intrínseca podem ocorrer devido a doenças sistêmicas, alterações na formação do dente, trauma dental, necrose pulpar, uso de fármacos, fluorose e pelo próprio processo de envelhecimento dental. Entre os fatores extrínsecos estão: tabagismo, medicamentos como a clorexidina, acúmulo de placa bacteriana, e principalmente, a ingestão de alimentos e bebidas que contenham corantes ${ }^{8}$.

Café, chá, sucos, vinho e bebidas ácidas têm potencial de coloração, que podem manchar ou descolorir a superfície do esmalte clareado. Alguns deles são soluções ácidas que podem aumentar a desmineralização, enquanto outras contêm etanol ou pigmentos. É possível que a superfície do esmalte clareado seja mais suscetível a pigmentação ${ }^{9}$.

Nesse contexto, graças ao alto apelo estético atual, tem estado presente na maioria das dietas as bebibas fitness, que visam desintoxicação do organismo e promoção de emagrecimento, nas quais podemos encontrar muitos componentes com alto teor de acidez como limão, abacaxi, couve entre outros e pigmentantes como beterraba, cenoura, açaí, podendo causar também manchamento e danos ao esmalte.

Com relação aos resultados satisfatórios os profissionais têm se mostrado preocupados com os efeitos do peróxido de carbamida e peróxido de hidrogênio de branqueamento quando entram em contato com os tecidos da cavidade bucal, e tal procedimento tem estimulado estudos de avaliação das possíveis reações adversas produzidas durante e após o branqueamento 5 .

Diante do exposto, e percebendo- se o momento em que vivemos onde o movimento fitness tornou- se imperialista na busca pela melhor estética corporal no consumo de bebidas tipo detox. A investigação do processo de alterações de cor no esmalte humano quando clareado torna-se fundamental no desenvolvimento de protocolos que possibilitem ou auxiliem a manutenção estética e maior longevidade clínica do efeito do clareamento. O propósito deste estudo foi avaliar o efeito de bebidas fitness sobre a alteração de cor e a rugosidade superficial do esmalte dental clareado.

\section{MATERIAL E MÉTODO}

\section{Confecção de corpos de prova}

- Blocos de esmalte

Cem dentes bovinos extraídos foram devidamente limpos com curetas periodontais (Dental DuflexLtda), recebendo, em seguida, polimento com pedra pomes e água, com auxílio de escova tipo Robinson (KG Sorensen Ind. e Com. Ltda), montados em baixa rotação. Os dentes foram armazenados em solução de cloramina $0,5 \%$ durante uma semana e após este período, em água mineral em temperatura ambiente, até o momento de sua utilização. Em seguida, suas raízes foram removidas e descartadas, sendo confeccionados fragmentos da face vestibular dos dentes com dimensões $5 \times 5 \mathrm{~mm}$.

$\mathrm{Na}$ sequencia, as superfícies de esmalte foram planificadas manualmente em lixas abrasivas de óxido de alumínio de granulações 400, 600 e 1200 refrigeradas com água, sem pressão digital, passando por ultra-som 2210 (Branson) 2 minutos entre uma lixa e outra e ao final do processo, para uma limpeza adequada das amostras, a fim de evitar resíduos de granulação da lixa antecessora. Após a obtenção de uma superfície lisa e polida, os corpos-de-prova foram limpos por 10 minutos com solução detergente neutra em cuba de ultrassom.

\section{- Grupamentos experimentais}

Após as análises iniciais, todos os dentes foram divididos em 10 grupos de estudo $(n=10)$ e receberam os seguintes tratamentos conforme o Protocolo (clareados e controle) e a solução corante (Quadro 1).

\begin{tabular}{|c|c|}
\hline Tratamento Clareador & Solução Corante \\
\hline $\begin{array}{c}\text { Espécimes permaneceram } \\
\text { armazenados em saliva artificial } \\
\text { durante todo o tempo } \\
\text { experimental em que não } \\
\text { estavam nas soluções corantes- } \\
\text { Não foram clareados (Controle) }\end{array}$ & $\begin{array}{c}\text { Grupo I- Suco de açaí }(\mathrm{n}=10) \\
\text { Grupo II- Suco Detox rosa }(\mathrm{n}=10) \\
\text { Grupo III- Suco Detox verde }(\mathrm{n}=10) \\
\text { Grupo IV-Suco Detox amarelo }(\mathrm{n}=10) \\
\text { Grupo V- Água mineral }(\mathrm{n}=10)\end{array}$ \\
\hline $\begin{array}{l}\text { Clareador Whiteness HP } \\
\text { Automixx (FGM Produtos } \\
\text { Odontológicos), à base de } \\
\text { peróxido de hidrogênio à 35\% }\end{array}$ & $\begin{array}{c}\text { Grupo VI- Suco de açaí }(n=10) \\
\text { Grupo VII- Suco Detox rosa }(n=10) \\
\text { Grupo VIII- Suco Detox verde }(n=10) \\
\text { Grupo IX- Suco Detox amarelo }(n=10) \\
\text { Grupo X- Água mineral }(n=10)\end{array}$ \\
\hline
\end{tabular}

Fonte: Dados da pesquisa 


\section{Clareamento}

Cem corpos de prova foram confeccionados para este estudo a partir do corte dos dentes apresentando como dimensões $5 \mathrm{mmx} 5 \mathrm{~mm}$. Para a realização do experimento os blocos de esmalte foram divididos de acordo com os grupos clareados e não clareados, totalizando 50 amostras de esmalte para cada Protocolo (grupos clareados e não clareados).

- Protocolo de envelhecimento em sucos Detox dos grupos não clareados

No Grupo I o processo de envelhecimento artificial semanal consistiu da imersão diária das amostras durante 1 hora em suco de açaí, em estufa, a $37^{\circ} \mathrm{C}$ seguida pela escovação com escova Soft Slim Black $®$ e creme dental Colgate total12, por meio da técnica de escovação de fones, sendo as amostras armazenadas em saliva artificial até a próxima imersão no dia seguinte. Este simulou uma situação clínica na qual o paciente, mantém sua rotina diária de ingestão de bebidas fitness como, neste caso, o suco de açaí.

Nos Grupos II, III, IV e $\mathrm{V}$ os dentes receberam os mesmos tratamentos do Grupo I, mas sofreram o envelhecimento, por 1 hora em suco detox rosa, suco detox verde, suco detox amarelo e água mineral, respectivamente.

Todos os dentes foram escovados por única pessoa para padronização, o método foi o de fones com escova dental Soft Slim Black® e creme dental Colgate total12.

\section{- Protocolo de envelhecimento em sucos Detox dos} grupos clareados

No Grupo VI os espécimes ficaram armazenados em eppendorfs e foram recobertos com Dessensibilize 0,2\%(FGM Produtos Odontológicos) por 10 minutos seguido da aplicação do produto a base de peróxido de hidrogênio a 35\% Whiteness HP Automixx(FGM Produtos Odontológicos), permanecendo em contato com o gel clareador por 15 minutos cada sessão semanal (total de 3 semanas); sendo que após cada sessão, os dentes receberam aplicação de flúor neutro e após 30 minutos, sofreram envelhecimento artificial em suco de açaí a $37^{\circ} \mathrm{C}$ por 1 hora sendo, então, limpos por meio de escova Soft Slim Black ${ }^{\circledR}$ e creme dental Colgate total12, por meio da técnica de escovação de Fones; sendo então, armazenados em saliva artificial até a próxima sessão de clareamento. $\mathrm{O}$ processo de envelhecimento artificial semanal (enquanto aguarda- se a próxima sessão de clareamento) ainda contou com a imersão diária das amostras durante 1 hora em suco de açaí, em estufa, a $37^{\circ} \mathrm{C}$ e seguida pela escovação como acima descrito, sendo as amostras armazenadas em saliva artificial até a próxima imersão no dia seguinte. Este simulou uma situação clínica na qual o paciente, que está sendo submetido a um tratamento clareador em consultório totalizando 3 semanas de Tratamento, continua com a sua rotina diária de ingestão de bebidas fitness como, neste caso, o suco de açaí, enquanto aguarda a próxima sessão de clareamento semanal.

Nos Grupos VI, VIII, IX e $\mathrm{X}$ os dentes receberam os mesmos tratamentos do Grupo VI, mas sofreram o envelhecimento, por 1 hora em suco detox rosa, suco detox verde, suco detox amarelo e água mineral, respectivamente. As soluções corantes e a composição das mesmas estão descritos no Quadro 2.

Quadro 2. Composição das bebidas utilizadas
\begin{tabular}{|c|c|}
\hline Suco Detox & Composição \\
\hline Suco de açaí & Açaí e laranja \\
\hline Suco Detox rosa & Cenoura, beterraba, água mineral \\
\hline Suco Detox amarelo & Abacaxi, hortelã, água de coco e raspas de limão \\
\hline Suco Detox verde & Abacaxi, couve, maçã, limão, água de coco, gengibre \\
\hline
\end{tabular}
Fonte: Dados da pesquisa

Todos os dentes foram escovados por única pessoa para padronização, o método foi o de Fones com escova dental Soft Slim Black® e creme dental Colgate total12.

\section{- Armazenamento das amostras durante o experimento}

Os dentes foram armazenados em estufa a $37^{\circ} \mathrm{C}$ durante todo o período experimental simulando condições bucais em recipientes individuais (eppendorfs de $2 \mathrm{~mL}$ ) e personalizados (numerados/identificados) para cada grupo. $\mathrm{O} \mathrm{Ph}$ de todas as soluções utilizadas nesta pesquisa foram mensurados através de pHmetro.

Quadro 3. $\mathrm{pH}$ do Gel clareador e das soluções corantes utilizadas no experimento

\begin{tabular}{|c|c|}
\hline Solução & pH \\
\hline Saliva Artificial & 6,59 \\
\hline Suco de Acaí & 4,05 \\
\hline Suco Detox Rosa & 6,80 \\
\hline Suco Detox Verde & 3,21 \\
\hline Suco Detox Amarelo & 4,14 \\
\hline Água Mineral & 7,62 \\
\hline Fonte: Dados da pesquisa
\end{tabular}

- Avaliação da cor e envelhecimento artificial em soluções corantes

Todos os corpos-de-prova foram submetidos a uma análise cromática inicial, por meio de um colorímetro EasyShade, com a avaliação de cor calculada através do Sistema CIE L*a*b*, estabelecido pela Comission Internacionale de I'Eclairaga - CIE. Este consiste de dois eixos a* e $b^{*}$, que possuem ângulos retos e representam a dimensão da tonalidade ou cor $\left(\mathrm{a}^{*}\right.$ : proporção vermelho-verde; $b^{*}$ : proporção amarelo-azul). O terceiro eixo é o brilho, representado pela letra L*. Este é perpendicular ao plano $\mathrm{a}^{*} \mathrm{~b}^{*}$. Com este sistema, qualquer cor pode ser especificada com as coordenadas $\mathrm{L}^{*}, \mathrm{a}^{*}, \mathrm{~b}^{*}{ }^{11,12}$.

Após a análise cromática inicial, todos os corpos-de-prova foram levados ao processo de 
envelhecimento artificial em bebidas fitness: Suco de açaí, Suco detox verde, Suco detox rosa, Suco detox amarelo além de um grupo controle (água mineral).

$\mathrm{O}$ método de envelhecimento consistiu na imersão das amostras nas soluções corantes durante 3 semanas, sendo que as mesmas foram trocadas diariamente. A alteração de cor foi determinada pela diferença $(\Delta \mathrm{E})$ entre as coordenadas obtidas das amostras antes e após o procedimento de envelhecimento.

A mudança total de cor, $\Delta \mathrm{E}$, é comumente usada para representar uma diferença de cor e é calculada a partir da fórmula:

$$
\Delta \mathrm{E}=\sqrt{ }(\Delta \mathrm{L} *)^{2}+\left(\Delta \mathrm{a}^{*}\right)^{2}+\left(\Delta \mathrm{b}^{*}\right)^{2}
$$

Os corpos-de-prova foram armazenados em estufa a $37^{\circ} \mathrm{C}$ durante todo o período experimental.

Avaliação da rugosidade

A análise da rugosidade da superfície foi realizada antes (T1) e depois (T2) do processo de envelhecimento artificial nas bebidas fitness descrita acima utilizando rugosímetro Hommel Etamic W10 (JENOPTIK Industrial Metrology Germany $\mathrm{GmbH}$ ). Este instrumento é equipado com uma agulha de diamante com um raio de $2 \mathrm{~mm}$. Para fazer medições da rugosidade, a agulha movida a uma velocidade constante de $0,5 \mathrm{~mm} / \mathrm{s}$ com uma carga de $0,7 \mathrm{mN}$. O valor de corte foi definido em $0.25 \mathrm{~mm}$. A rugosidade da superfície foi caracterizada pela rugosidade média (Ra) que é o valor da média aritmética de todas as distâncias absolutas do perfil de rugosidade a partir da linha de centro, dentro do comprimento de medida. Valores de Ra para cada amostra foram tomados ao longo do diâmetro sobre um comprimento padrão de $0,25 \mathrm{~mm}$. Três traços foram registrados em cada amostra em três diferentes locais, paralelo, oblíquo e perpendicular.

Análise estatística

Os dados de alteração de cor $(\Delta E)$ e rugosidade ( $\mathrm{Ra}$ ) dos blocos de esmalte submetidos a bebidas fitness foram tabulados e submetidos a uma análise exploratória e por intermédio dos testes de Shapiro Wilk e Bartlett foram verificadas a normalidade dos resíduos e a homogeneidade das variâncias, respectivamente. Posteriormente, realizou-se a Análise de Variância dois fatores, com pós-teste de Tukey. A análise estatística foi realizada com auxílio do software $\mathrm{R}$, tendo sido considerado nível de significância de 5\%.

\section{RESULTADOS}

Não foi observada diferença estatisticamente significante na rugosidade das amostras independente da realização de clareamento ou não e das bebidas detox utilizadas neste estudo $(\mathrm{p}=0.067)$ (Tabela 1$)$. Alteração de cor

$\mathrm{Na}$ comparação entre os sucos Detox avaliados neste estudo na Tabela 2 foi constatado que, nos grupos das amostras de esmalte que foram clareadas, os sucos Detox Amarelo e Rosa (p>0,05), sem diferença estatisticamente significante para água, apresentaram maior alteração de cor comparativamente as amostras imersas em Suco Detox Verde e Suco de Açai $(\mathrm{p}<0,05)$ os quais apresentaram menor alteração de cor após o clareamento.

Já entre os grupos de amostras de esmalte que não foram clareadas previamente, os Sucos Detox Rosa, Suco de Açai e Suco Detox Verde apresentaram maior alteração de cor $(p>0,05)$ comparativamente as amostras imersas em Suco Detox Amarelo e Água ( $\mathrm{p}<0,05)$ (Tabela 2).

\begin{tabular}{|c|c|c|c|c|}
\hline $\begin{array}{l}\text { Suco } \\
\text { Detox }\end{array}$ & $\begin{array}{c}\text { Clareado } \\
\text { Inicial }\end{array}$ & $\begin{array}{c}\text { Clareado } \\
\text { Final }\end{array}$ & $\begin{array}{c}\text { Não Clareado } \\
\text { Inicial }\end{array}$ & $\begin{array}{c}\text { Não Clareado } \\
\text { Final }\end{array}$ \\
\hline Agua & $3.83(1.42)$ & $4.41(2.12)$ & $4.96(1.65)$ & $3.22(1.77)$ \\
\hline Açai & $2.58(1.13)$ & $2.25(1.57)$ & $3.13(1.07)$ & $2.99(1.65)$ \\
\hline DTXAm & $3.64(1.55)$ & $5.53(3.55)$ & $3.16(1.20)$ & $3.67(2.38)$ \\
\hline DTXRs & $3.49(1.85)$ & 3.48 (1.99) & $2.86(1.36)$ & $3.68(2.84)$ \\
\hline DTXVr & $5.86(3.58)$ & $4.62(3.41)$ & $4.05(2.0)$ & $3.68(2.85)$ \\
\hline
\end{tabular}

Tabela 2. Alteração de cor $(\Delta \mathrm{E})$ em função do clareamento e sucos Detox

\begin{tabular}{l|l|l}
\hline \multirow{1}{*}{ Sucos Detox } & \multicolumn{2}{c}{ Clareamento } \\
\cline { 2 - 3 } & \multicolumn{1}{|c}{ Clareado } & \multicolumn{1}{c}{ Não Clareado } \\
\hline Água & $17.19(2.08) \mathrm{Aa}$ & $3.37(1.67) \mathrm{Bc}$ \\
Açaí & $7.25(1.90) \mathrm{Bb}$ & $14.41(2.30) \mathrm{Aab}$ \\
DTXAm & $13.87(3.29) \mathrm{Aa}$ & $6.35(2.83) \mathrm{Bc}$ \\
DTXRs & $14.57(5.30) \mathrm{Ba}$ & $18.06(4.68) \mathrm{Aa}$ \\
DTXVr & $6.97(2.18) \mathrm{Bb}$ & $12.30(2.51) \mathrm{Ab}$ \\
\hline \multicolumn{1}{|c|}{ Médias seguidas de letras distintas (minúsculas na vertical e maiúsculas na } \\
horizontal) diferem entre $\mathrm{si}(\mathrm{p} \leq 0,05)$
\end{tabular}

Na Tabela 3, analisando os valores de $\mathrm{L}^{*}$ com relação a luminosidade para os grupos que permaneceram em água, independente se foi ou não realizado clareamento, não foi observada diferença estatisticamente significante para o $\Delta \mathrm{L}$, sem diferença estatisticamente significante para as amostras que foram clareadas e imersas em suco detox amarelo. As amostras que permaneceram em suco detox rosa, independente se recebeu clareamento ou não, apresentaram $\Delta \mathrm{L}$ mais elevado indicando maior perda de luminosidade.

Tabela 3. $\Delta \mathrm{L}$ em função do Clareamento e Sucos Detox

\begin{tabular}{l|l|l|l|l|l|l}
\hline \multirow{2}{*}{} & \multicolumn{5}{c|}{ L } \\
\cline { 2 - 8 } & \multicolumn{2}{|c|}{$\mathbf{L o}^{-}$} & \multicolumn{2}{c}{$\mathbf{L}_{\mathbf{f}}$} & \multicolumn{2}{c}{$\Delta \mathbf{L}$} \\
\hline Açaí clareado & 90.03 & $(3.40)$ & 85.08 & $(5.05)$ & -4.95 & $(3.05)^{\mathrm{ab}}$ \\
Açaí não clareado & 92.45 & $(3.92)$ & 82.17 & $(2.38)$ & -10.28 & $(3.18)^{\mathrm{bc}}$ \\
Detox amarelo clareado & 91.46 & $(5.33)$ & 91.53 & $(5.18)$ & 0.07 & $(8.72)^{\mathrm{a}}$ \\
Detox amarelo não clareado & 92.28 & $(4.28)$ & 89.04 & $(5.24)$ & -3.24 & $(4.34)^{\mathrm{ab}}$ \\
Detox rosa clareado & 90.24 & $(7.07)$ & 74.56 & $(13.34)$ & -15.68 & $(8.51)^{\mathrm{c}}$ \\
Detox rosa não clareado & 88.60 & $(6.15)$ & 72.30 & $(3.28)$ & -16.30 & $(7.41)^{\mathrm{c}}$ \\
Detox verde clareado & 90.51 & $(9.31)$ & 86.32 & $(7.81)$ & -4.19 & $(2.69)^{\mathrm{ab}}$ \\
Detox verde não clareado & 93.89 & $(3.16)$ & 85.28 & $(5.49)$ & -8.61 & $(4.83)^{\mathrm{bc}}$ \\
Água clareado & 92.30 & $(4.48)$ & 92.62 & $(2.67)$ & 0.32 & $(5.88)^{\mathrm{a}}$ \\
Água não clareado & 84.67 & $(14.93)$ & 84.81 & $(13.39)$ & 0.14 & $(2.56)^{\mathrm{a}}$ \\
\hline
\end{tabular}

Nesta Tabela 4, foi evidente a diferença observada entre os valores de $\mathrm{B}^{*}$ final e $\mathrm{B} *$ inicial para todos os grupos de amostras que foram submetidas ao clareamento apresentando valores de $\triangle \mathrm{B}$ negativos. As amostras que foram clareadas $\mathrm{e}$ permaneceram em água apresentaram $\Delta \mathrm{B}$ de $-16,04$ comparativamente com as amostras que permaneceram em água e não foram clareadas (grupo 
controle) que, por outro lado, apresentaram $\Delta \mathrm{B}$ de 0,65 . Já as amostras que não foram clareadas e foram imersas em sucos detox apresentaram valores de $\Delta \mathrm{B}$ positivos. As amostras imersas em suco de açaí apresentaram maior $\Delta \mathrm{B}$ sem diferença estatisticamente significante para os sucos detox verde e detox rosa, os quais apresentaram valores intermediários, comparativamente ao suco detox amarelo que apresentou $\Delta \mathrm{B}$ de 0,41 .

Tabela 4. $\Delta \mathrm{B}$ em função do Clareamento e Sucos Detox

\begin{tabular}{|c|c|c|c|c|c|c|}
\hline \multirow{3}{*}{ Açaí clareado } & \multicolumn{6}{|c|}{ B } \\
\hline & \multicolumn{2}{|c|}{$\mathbf{B}_{\mathbf{o}}$} & \multicolumn{2}{|c|}{$\mathbf{B}_{\mathbf{f}}$} & \multicolumn{2}{|c|}{$\Delta \mathbf{B}$} \\
\hline & 23.55 & $(5.19)$ & 20.59 & $(4.39)$ & -2.96 & $(2.96)^{\mathrm{bc}}$ \\
\hline Açaí não clareado & 25.49 & (10.15) & 28.86 & $(5 \cdot 39)$ & $3 \cdot 37$ & $(9.03)^{a}$ \\
\hline Detox amarelo clareado & 30.23 & $(3.19)$ & 19.62 & $(5 \cdot 37)$ & -10.61 & $(4.42)$ de \\
\hline Detox amarelo não clareado & 29.31 & $(5.61)$ & 29.72 & $(7.16)$ & 0.41 & $(4.22)^{b c}$ \\
\hline Detox rosa clareado & 30.87 & $(6.86)$ & 26.35 & $(5.81)$ & $-4.5^{2}$ & $(4.99)^{\mathrm{cd}}$ \\
\hline Detox rosa não clareado & 27.83 & $(4.76)$ & 30.73 & $(5.66)$ & 2.90 & $(6.38)^{a b}$ \\
\hline Detox verde clareado & 30.54 & $(3.70)$ & 26.64 & $(4.13)$ & -3.90 & $(3.58)^{c}$ \\
\hline Detox verde não clareado & 30.07 & $(4 \cdot 34)$ & 33.54 & $(4.62)$ & 3.47 & $(6.92)^{a b}$ \\
\hline Água clareado & 32.85 & $(3.72)$ & 16.81 & $(2.84)$ & -16.04 & $(2.11)^{\mathrm{e}}$ \\
\hline Água não clareado & 32.94 & $(7.53)$ & 32.29 & $7.25)$ & -0.65 & $(2.58))^{b c}$ \\
\hline
\end{tabular}

\section{DISCUSSÃO}

A exigência com relação à estética tem crescido em todas as áreas, não sendo a Odontologia uma exceção. Há algum tempo era aceitável, por exemplo, apresentar dentes amarelados ou com pequenas alterações na cor, não sendo considerado um fator desarmônico ${ }^{1,17}$. O dente escurecido é assim visualizado em função da maior absorção de luz, que ocorre pela presença de cadeias moleculares longas e complexas no interior da estrutura dental. Já o dente que possui uma coloração menos saturada absorve menos luz, gerando percepção óptica de uma superfície mais clara, devido a maior reflexão da luz.

Neste contexto, o clareamento dental tem sido uma opção conservadora, como medida corretiva para o tratamento das pigmentações dentárias ${ }^{1,3,15}$ por influenciar diretamente a reflexão da luz irá determinar o valor e o croma da estrutura dentária respectivamente, baseado na variação do brilho e no seu grau de saturação. Assim, o uso generalizado dos agentes clareadores para fins estéticos torna necessária a escolha adequada de materiais com mais rigor nas técnicas seguindo de maneira criteriosa os protocolos clareadores que promovam resultados desejados com efeitos mínimos sobre o esmalte dental $^{18}$.

Atualmente o uso dos agentes clareadores está tão difundido que muitas pessoas fazem seu uso aleatoriamente, sem a devida supervisão do cirurgião dentista, o que explica o grande volume de pesquisas que busquem investigar possíveis efeitos indesejáveis sobre a estrutura dentária. Perda mineral, aumento da rugosidade superficial, diminuição da microdureza tem sido continuamente reportados em vários estudos ${ }^{1,15,21,23-25}$.

Este estudo avaliou o efeito do clareamento com peróxido de hidrogênio utilizado em técnica de consultório sobre a rugosidade superficial e alteração de cor da estrutura dentária mantida sob envelhecimento artificial em bebidas fitness que apresentavam baixo $\mathrm{pH}$ devido a sua própria composição. De acordo com os resultados obtidos não foi observada diferença estatisticamente significante na porcentagem de aumento da rugosidade das amostras independente da realização de clareamento ou não e das bebidas detox utilizadas neste estudo.

Apesar da maioria dos trabalhos mostrarem um aumento na porcentagem de aumento da rugosidade após a realização do clareamento tal fato não foi observado nesta investigação. Esse resultado pode ser atribuído à capacidade da saliva em prevenir tais alterações na estrutura dentária ${ }^{22}$ e sugere a reversibilidade das alterações relacionadas ao clareamento quando utilizada durante todo o tempo experimental ${ }^{12}$ simulando condições bucais reais.

Dessa forma, sugere- se que as sessões mais breves (15 minutos) de clareamento com peróxido de hidrogênio a $35 \%$ semanais associado ao fato do dente ter sido mantido em saliva artificial durante todo o período experimental favoreceu a manutenção de sua rugosidade superficial. Associado a isso, neste estudo, todas as amostras foram submetidas ao processo de escovação e uso de dentifrício fluoretado, o que pode ter contribuído ainda mais para a remineralização ${ }^{5,33}$ do esmalte juntamente com o uso da saliva artificial ${ }^{5,34}$. Além disso, o uso das bebidas detox foi realizado seguindo recomendação de nutricionista, simulando portanto situações reais, sendo a superfície de esmalte exposta as bebidas detox apenas pelo tempo de 60 minutos diariamente, tempo em média que uma pessoa realiza seu desjejum ${ }^{5}$ sendo então escovados e mantidos em saliva artificial pelas seguintes 23 horas até o próximo desafio de envelhecimento.

O Sistema CIE L*A*B* é uma medição de cor tridimensional e é amplamente utilizado para quantificar e descrever a cor, sendo três coordenadas onde $\mathrm{L}^{*}$ representa a luminosidade que varia do preto ao branco, $\mathrm{A}^{*}$ é a medida do croma no eixo vermelho- verde, $\mathrm{B}^{*}$ é a medida do croma de amarelo a azul. Esse eixo $\mathrm{B}^{*}$ é a medida de amarelado (b positivo) e do azulado (b negativo) ${ }^{28}$. Valores positivos para o eixo $\mathrm{B}^{*}$ são mais amarelos, enquanto valores negativos são mais azuis ${ }^{5}$. Quando o clareamento dental é realizado através do esmalte ocorre a difusão do peróxido de hidrogênio através do esmalte para o interior do dente $e^{15,16,19,20}$ favorecendo o processo de reflexão de luz e elevando a luminosidade do substrato.

Neste trabalho, houve uma diferença estatística elevada entre as amostras que foram clareadas $(\Delta \mathrm{E}=17.19)$ e não clareadas $(\Delta \mathrm{E}=3,37)$, as quais foram submetidas a imersão em água mineral por 60 minutos diários. Analisando os valores de L* 
obtidos nesse estudo com relação a luminosidade para esses grupos que permaneceram em água não foi observada diferença estatisticamente significante para o $\Delta \mathrm{L}$ de ambos os grupos que explicasse essa diferença no $\Delta \mathrm{E}$. Por outro lado, foi evidente a diferença observada entre os valores de $\mathrm{B}^{*}$ final e $\mathrm{B}^{*}$ inicial para estes grupos, sendo que as amostras que foram clareadas e permaneceram em água apresentaram um valor de $\Delta \mathrm{b}$ de $-16,04$ contra os 0,65 das amostras que permaneceram em água e não foram clareadas (grupo controle). Os valores do eixo $\mathrm{B}^{*}$ são considerados as variáveis mais significativas nas pesquisas de branqueamento dental. Sabe- se que a obtenção de $\Delta \mathrm{B}$ negativo ao final do clareamento assegura que a coloração amarelada das amostras dentais foram direcionadas para os tons azulados, assinalando que ocorreu o branqueamento do substrato $^{5,35}$, corroborando com os resultados obtidos nesse estudo.

Além disso, foi verificada maior alteração de cor do substrato bovino clareado quando submetido a manchamento por 60 minutos diários durante 3 semanas nos sucos Detox Amarelo $(\Delta \mathrm{E}=13,87$ e $\mathrm{pH}$ $4,1)$ e Rosa $(\Delta \mathrm{E}=14,27$ e $\mathrm{pH} 6,8)$, comparativamente as amostras imersas em suco Detox Verde $(\Delta \mathrm{E}=6,97$ e pH 3,21) e Suco de Açaí ( $\Delta E=7,25$ e pH 4,05) os quais apresentaram menor alteração de cor após o clareamento. Nessa comparação entre os resultados das amostras que foram clareadas e imersas em sucos detox comparativamente com as amostras que foram submetidas a imersão em água mineral pode ser também explicada pelo $\Delta \mathrm{B}$ e $\Delta \mathrm{L}$ das amostras após o clareamento. Ocorreu um leve aumento na luminosidade das amostras que permaneceram em água durante todo o experimento independente se foram clareadas $(\Delta \mathrm{L}=0,32)$ ou não $(\Delta \mathrm{L}=0,14)$, porém sem diferença estatisticamente significante.

Com excessão das amostras que foram clareadas e permaneceram em suco detox amarelo $(\Delta \mathrm{L}=0,07)$, aquelas que foram imersas em sucos de açai $(\Delta \mathrm{L}=-4,95)$ e detox verde $(\Delta \mathrm{L}=-4,19)$ apresentaram perda de luminosidade, indicando o seu manchamento, e consequente escurecimento das amostras. As amostras que permaneceram em detox rosa apresentaram maior perda de luminosidade $(\Delta \mathrm{L}=$ $-15,68)$ corroborando com os valores de alteração de cor $\Delta \mathrm{E}$ obtidos para esse grupo $(\Delta \mathrm{E}=14,27)$.

Esses resultados podem ser explicados pela diferença no $\mathrm{pH}$, na composição dos sucos avaliados neste estudo e seu potencial corante. Em estudo realizado "in vitro" para avaliar o efeito de soluções ácida, neutra e alcalina, com pH 2, pH 7 e pH 12, mostrou que a intensidade e tipo de manchamento no esmalte dental foram influenciados pelo $\mathrm{pH}$ reduzido e o alimento corante onde ocorreu a dissolução do esmalte $^{28}$ corroborando com os resultados desse estudo. Apesar de não apresentar $\mathrm{pH}$ considerado ácido, o suco detox rosa, apresenta a baterraba em sua composição com partículas químicas que causam a pigmentação vermelha devido a combinação de dois tipos de pigmentos: betacianina (roxo) e betaxanatina (amarelo) o que pode explicar o maior manchamento e maior perda de luminosidade das amostras. Por outro lado o suco detox amarelo apresenta pH ácido por apresentar em sua composição alimentos extremamente ácidos como o limão, o abacaxi e o hortelã.

Alguns estudos relatam que bebidas ácidas são elevadamente pigmentantes causando manchamento e dissolução das estruturas dentárias duras $^{29-32}$. Estes autores através de suas pesquisas ${ }^{29-32}$ observaram que após imersão em suco cítricos, bebidas carbonatadas (refrigerantes), esportivas, cerveja e outras bebidas ácidas a diminuição da dureza do esmalte foi substancial (84\%), por esse motivo acredita-se que os pigmentos consumidos através da alimentação, durante $o$ período do clareamento, podem levar a uma pigmentação na estrutura do esmalte sendo que o tempo adequado da remineralização do esmalte dental pós clareamento para o consumo seguro de alimentos pigmentados ainda apresenta resultados adversos ${ }^{19-21}$ e controversos na literatura.

Apesar do manchamento evidente das amostras que foram imersas nos sucos detox e suco de açaí avaliados neste estudo, apresentando valores de $\Delta \mathrm{E}$ considerados altos e clinicamente visíveis e perceptíveis, foi possível constatar que todas as amostras que foram submetidas ao clareamento apresentaram valores de $\Delta \mathrm{B}$ negativos, indicando a diminuição de tons amarelos nos substratos, confirmando assim que houve o clareamento das amostras. Porém as amostras que estavam em água apresentaram maior clareamento $(\Delta \mathrm{B}=-16,04)$ comparativamente aquelas que estavam em detox amarelo $(\Delta \mathrm{B}=-10,61)$, suco de açai $(\Delta \mathrm{B}=-2,96)$, detox verde $(\Delta \mathrm{B}=-3,90)$ e detox rosa $(\Delta \mathrm{B}=-4,52)$.

Diante do exposto este trabalho buscou simular uma situação clínica o mais próximo do real e vida cotidiana de um paciente que está sob atendimento odontológico e acompanhamento de nutricionista com ingestão diária dos sucos detox durante o período de clareamento de consultório com Peróxido de Hidrogênio a $35 \%$. Tais hábitos demostraram promover o manchamento dos dentes além de diminuir a eficácia clareadora do gel clareador. Entretanto torna-se necessário mais estudos sobre a interferência da ingestão de bebidas detox e açaí durante o clareamento sobre as propriedades do substrato dental.

\section{CONCLUSÃO}

Não foi observada diferença estatisticamente significante na porcentagem de aumento da rugosidad e das amostras. Apresentaram maior alteração de cor, os substratos dentais clareados que foram submetidas 
a envelhecimento artificial em suco detox amarelo e s uco detox rosa. Entre os grupos que não foram clarea dos, os Sucos detox rosa, de açai e detox verde aprese ntaram maior alteração de cor.

\section{REFERÊNCIAS}

1. Attia ML, Gomes ACO, César ICR, Munin E, Aguiar FHB, Liporoni PCS. Avaliação da eficácia de clareamento e da susceptibilidade ao manchamento de blocos dentais humanos e bovinos submetidos a dois agentes pigmentantes. In: Anais do IX Encontro Latino Americano de Iniciação Científica e $\mathrm{V}$ Encontro Latino Americano de Pós-Graduação. João Pessoa: Universidade do Vale do Paraíba; 2005.

2. Ramos APB, Cesar ICR, Alves GL, Alves LP, Munin E, Rego MA, Liporoni PC. Avaliação do clareamento dental com peróxido de carbamida a $16 \%$, submetidos a diferentes pigmentos, através de análise de fotorreflectância e rugosidade. In: Anais do $\mathrm{X}$ Encontro Latino Americano de Iniciação Científica e VI Encontro Latino Americano de Pós-Graduação - Universidade do Vale do Paraíba. João Pessoa: Universidade do Vale do Paraíba; 2005.

3. Souto CMC. Avaliação da influência de ingestão de bebidas corantes em diferentes tempos na estabilidade do clareamento dental: análise de fotorreflectância. [dissertação]. Taubaté: Universidade de Taubaté; 2006.

4. Sundfeld RH. Clareamento de Dentes Vitais com Peróxido de Carbamida. Araçatuba: Unesp, 2013. Disponível em: http://www.foa.unesp.br/include/ arquivos/foa/restauradora/files/capituloclareamento-de-dentes-vitais-com-peroxido-decarbmida.pdf

5. Araújo LS, Santos PH, Anchieta RB, Catelan A, Fraga Briso AL, Fraga Zaze AC, Sundfeld RH. Mineral loss and color change of enamel after bleaching and staining solutions combination. J Biomed Opt. 2013;18(10):108004-6.

6. Anaraki SN, Shahabi S, Chiniforush N, Nokhbatolfoghahaei H, Assadian H, Yousefi B. Evaluation of the effects of conventional versus laser bleaching techniques on enamel microroughness. Lasers Med Sci. 2015; 30(3):1013-18.

7. Esberard RR, Consolaro A, Esberard RM, Bonetti I, Esberard RR.Efeitos das técnicas e dos agentes clareadores externos na morfologia da junção amelocementária e nos tecidos dentários que a compõem. Rev Dental Press Estét. 2004;1(1):58-72.

8. Rezende M, Cerqueira RR, Loguercio AD, Reis A, Kossatz S. Corantes com e sem açúcar versus efetividade do clareamento dental: estudo ex vivo. Rev Odontol Bras Central 2014;23(66):146-49.

9. Berger SB, Coelho AS, Oliveira VAP, Cavalli V, Giannini M. Enamel susceptibility to red wine staining after 35\% hydrogen peroxide bleaching. J. Appl. Oral Sci. 2008;16(3):201-4.

10. Whiteness. Clareador dental para uso em consultório somente uso profissional. Joenvile, SC: Dentscare; 2015. Disponível em: http://www.fgm.ind.br/site/wpcontent/uploads/2015/12/Whiteness-HP-manualde-info.pdf

11. Schulze KA, Marshall SJ, Gansky AS, Marshall GW. Color stability and hardness in dental composites after accelerated aging. Dent Mater. 2003;19(7):612-19.

12. Miranda TAM, Moura SK, Amorim VHO, Terada RSS, Pascotto RC. Influence of exposure time to saliva and antioxidant treatment on bond strength to enamel after tooth bleaching: an in situ study. J Appl Oral Science. 2013;21(6):567-74.

13. Tober T, Gilde H, Lenz P. Color stability of highly filled composite resin materials for facings. Dent Mater. 2001;17(1):87-94

14. Da Cunha FB, Rodrigues e Silva BH, Freitas De Paula BL, Alencar CM, de Albuquerque Jassé FF, Silva CM. Effect of high concentrated fluoridebased dentifrice on the hardness, roughness, and color of the bleached enamel. J Conserv Dent. 2018;21(4):433-37.

15. Carlos NR, Pinto A, Amaral FD, França F, Turssi $\mathrm{CP}$, Basting RT. Influence of staining solutions on color change and enamel surface properties during at-home and in-office dental bleaching: an in situ study. Oper Dent. 2019;44(6):595-608.

16. Attia ML, Cavalli V, do Espírito Santo AM, Martin AA, D'Arce MB, Aguiar FH et al. Effects of bleaching agents combined with regular and whitening toothpastes on surface roughness and mineral content of enamel. Photomed Laser Surg. 2015;33(7):378-83.

17. Liporoni PC, Souto CM, Pazinatto RB, Cesar IC, de Rego MA, Mathias $P$ et al. Enamel susceptibility to coffee and red wine staining at different intervals elapsed from bleaching: a photoreflectance spectrophotometry analysis. Photomed Laser Surg. 2010;28(Suppl 2):S105-9.

18. Al-Basher G, Al-Motiri H, Al-Farraj S, Al-Otibi $\mathrm{F}$, Al-Sultan N, Al-Kubaisi $\mathrm{N}$ et al. Chronic exposure to $35 \%$ carbamide peroxide tooth bleaching agent induces histological and hematological alterations, oxidative stress, and inflammation in mice. Environ Sci Pollut Res Int. 2019;26(17):17427-37.

19. Kothari S, Gray AR, Lyons K, Tan XW, Brunton PA. Vital bleaching and oral-health-related quality of life in adults: A systematic review and metaanalysis. J Dent. 2019;84:22-29.

20. Joiner A. Tooth colour and whiteness: a review of the literature. J Dent. 2017;32(Suppl 1):3-12.

21. Attin T, Manolakis A, Buchalla W, Hannig C. Influence of tea on intrinsic colour of previously 
bleached enamel. J Oral Rehabil. 2003; 30(5):488-94.

22. Attin T, Schmidlin PR, Wegehaupt F, Wiegand A. Influence of study design on the impact of bleaching agents on dental enamel microhardness: a review. Dent Mater. 2009;25(2):143-57.

23. Pinto CF, Oliveira R, Cavalli V, Gianninni M. Peroxide bleaching agent effects on enamel surface microhardness, roughness and morphology. Braz Oral Res. 2004;18(4):306-11.

24. Potocnik I, Kosec L, Gaspersic D. Effect of $10 \%$ carbamide peroxide bleaching gel on enamel microhardness, microstructure, and mineral content. J Endod. 2000;26(4):203-6.

25. Torres CR, Koga AF, Borges AB. The effects of anti-oxidant agents as neutralizers of bleaching agents on enamel bond strength. Braz J Oral Sci. 2006;5(16):971-76.

26. Rezende M, Loguercio AD, Reis A, Kossatz. Clinical Effects of exposure to coffe during athome vital bleaching. Oper Dent. 2013; 38(6):E229-36.

27. Mori AA, Lima FF, Benetti AR, Terada RS, Fujimaki M, Pascotto RC. Susceptibility to coffee staining during enamel remineralization following the in-office bleaching technique: an in situ assessment. J Esthet Restor Dent. 2015; 28(Suppl 1):23-31.

28. Azer SS, Hague AL, Johnston WM. Effect of $\mathrm{pH}$ on tooth discoloration from food colorant in vitro. J Dent. 2010;38(Suppl 2):e106-9.

29. Al-Dlaigan YH, Shaw L, Smith A. Dental erosion in a group of British 14-year-old school children. Part II: Influence of dietary intake. Br Dent J. 2001;190(5):258-61.

30. Prati C, Montebugnoli L, Suppa P, Valdre` G, Mongiorgi R. Permeability and morphology of dentin after erosion induced by acidic drinks. J Periodontol. 2003;74(4):428-36.

31. Owens BM, Kitchens M. The erosive potential of soft drinks on enamel surface substrate: an in vitro scanning eléctron microscopy investigation. J Contemp Dental Pract. 2007;8(7):11-20.

32. Ren YF, Amin A, Malmstrom H. Effects of tooth whitening and orange juice on surface properties of dental enamel. J Dent. 2009;37(6):424-31.

\section{CONFLITO DE INTERESSES}

Os autores declaram não haver conflitos de interesse.

\section{AUTOR PARA CORRESPONDÊNCIA}

\section{Larissa Sgarbosa de Araújo Matuda}

larissa_sna@yahoo.com.br

Submetido em 10/06/2019

Aceito em 20/04/2020 